\title{
Adenovirus-mediated P311 ameliorates renal fibrosis through inhibition of epithelial-mesenchymal transition via TGF-ß31-Smad-ILK pathway in unilateral ureteral obstruction rats
}

\author{
FANG-HUA QI ${ }^{1}$, PING-PING CAI $^{1}$, XIANG LIU $^{2}$ and GUO-MIN SI ${ }^{1}$ \\ ${ }^{1}$ Department of Traditional Chinese Medicine and ${ }^{2}$ Department of Nephrology, \\ Shandong Provincial Hospital Affiliated to Shandong University, Jinan, Shandong 250021, P.R. China
}

Received December 29, 2016; Accepted February 6, 2018

DOI: $10.3892 / \mathrm{ijmm} .2018 .3485$

\begin{abstract}
Epithelial-mesenchymal transition (EMT) is a critical step and key factor during renal fibrosis. Preventing renal tubular EMT is important for delaying the progression of chronic kidney disease (CKD). P311, a highly conserved 8-kDa intracellular protein, has been indicated as an important factor in myofibroblast transformation and in the progression of fibrosis. However, the related studies on P311 on renal fibrosis are limited and the mechanisms of P311 in the progression of renal tubulointerstitial fibrosis remain largely unknown. In the present study, we examined the effect of P311 on transforming growth factor- $\beta 1$ (TGF- $\beta 1$ )-mediated EMT in a rat model of unilateral ureteral occlusion (UUO) renal fibrosis. The recombinant adenovirus p311 (also called Ad-P311) was constructed and transferred it into UUO rats, the preventive effect and possible mechanism of P311 on TGF- $\beta 1$-mediated EMT were explored. The UUO model was established successfully and Ad-P311 was administered into UUO rats each week for 4 weeks, then the serum levels of $\mathrm{Cr}$, blood urea nitrogen (BUN) and albumin (ALB) were evaluated. H\&E staining and Masson staining were performed to observe the pathological changes of kidneys. Immunohistochemical staining and western blot analysis were used to examine the EMT markers [E-cadherin and $\alpha$-smooth muscle actin ( $\alpha$-SMA)], and signal transducers (p-Smad2/3 and Smad7). Integrin linked kinase (ILK) as a
\end{abstract}

Correspondence to: Dr Guo-min Si, Department of Traditional Chinese Medicine, Shandong Provincial Hospital Affiliated to Shandong University, 324 Jingwuweiqi Road, Jinan, Shandong 250021, P.R. China

E-mail: sunsmile_sd@sina.com

Abbreviations: EMT, epithelial-mesenchymal transition; CKD, chronic kidney disease; UUO, unilateral ureteral occlusion; $\alpha$-SMA, $\alpha$-smooth muscle actin; ILK, integrin linked kinase; TGF- $\beta 1$, transforming growth factor- $\beta 1$

Key words: renal fibrosis, P311, epithelial-mesenchymal transition, transforming growth factor- $\beta 1$-Smad-integrin linked kinase pathway, unilateral ureteral occlusion rats keyintracellular mediator that controls TGF- $\beta 1$-mediated-EMT was also assayed by western blot analysis. The results showed that P311 could alleviate renal tubular damage and interstitial fibrosis improving Cr, BUN and ALB serum levels in UUO kidneys. Furthermore, P311 attenuated TGF- $\beta 1$-mediated EMT through Smad-ILK signaling pathway with an increase in $\alpha$-SMA, pSmad $2 / 3$ and ILK expression, and a decrease in E-cadherin and Smad7 expression in UUO kidneys. In conclusion, P311 may be involved in the pathogenesis of renal fibrosis by blocking TGF- $\beta 1$-mediated EMT via TGF- $\beta 1$-Smad-ILK pathway in UUO kidneys. P311 may be a novel target for the control of renal fibrosis and the progression of CKD.

\section{Introduction}

Chronic kidney disease (CKD) has become a major public health concern worldwide with higher morbidity and mortality (1). Renal fibrosis is generally recognized as the most prominent feature of CKD and the final common pathway leading to end-stage renal failure (2). The pathological changes associated with renal fibrosis mainly involve the deposition of extracellular matrix (ECM) which is thought to be produced by myofibroblasts (3). Epithelial-mesenchymal transition (EMT) is a complex process in which differentiated epithelial cells undergo a phenotypic conversion that gives rise to the matrixproducing fibroblasts and myofibroblasts (4). In the process of EMT, epithelial cells degrade the basement membrane to become migratory, with several epithelial markers such as E-cadherin, cytokeratin 18 (CK18) loss and mesenchymal markers such as $\mathrm{N}$-cadherin, fibronectin and $\alpha$-smooth muscle actin $(\alpha$-SMA) acquired $(5,6)$. EMT has been proved to be a critical step in the pathogenesis and progression of tubulointerstitial fibrosis, whereby renal tubular epithelial cells change phenotypically and functionally into myofibroblasts (7). Therefore, it is important to prevent tubular epithelial cells from undergoing EMT to prevent renal fibrosis.

As is known, chronic inflammation is acknowledged to be pivotal in the development and progression of CKD (8). When kidneys are exposed to inflammatory stimuli, the evaluated release of transforming growth factor- $\beta 1$ (TGF- $\beta 1$ ) eventually activates EMT of renal tubular cells (9). TGF- $\beta 1$, a well-known master cytokine/growth factor, is considered as an important 
well-established regulator of EMT. It could effectively regulate the transdifferentiation of tubular epithelial cells into myofibroblasts in renal fibrosis primarily via Smad-dependent pathway $(10,11)$. Upon TGF- $\beta 1$ binding to its receptors, Serine/ Threonine kinases are activated and induce phosphorylation of Smad2/Smad3, then phosphorylated Smad2/3 partner with Smad4 translocate into the nucleus where they regulate the transcription of the target genes responsible for EMT (10). Integrin linked kinase (ILK) is an intracellular serine/threonine kinase involved in cell-matrix interactions. It is shown to be a key intracellular mediator that controls TGF- $\beta 1$-induced-EMT in renal tubular epithelial cells $(11,12)$. Although the involvement of ILK in tubular EMT has been established based on several lines of evidence, intriguingly, many components of ILK signaling, including ILK and $\beta 1$-integrin are induced simultaneously by TGF- $\beta 1$ in a Smad-dependent manner $(4,12)$. Therefore, it is widely accepted that TGF- $\beta 1$ plays an important role in promoting renal tubular EMT.

$\mathrm{P} 311$, as a binding protein of the TGF- $\beta 1$ latency associated protein, is an $8-\mathrm{kDa}, 68$-amino acid, intracellular polypeptide that is highly conserved across species and expressed in brain, smooth muscle, regenerating tissues, and malignant glioblastomas (13-19). It has been shown to be of importance in the process of myofibroblast differentiation and fibrosis with the functions of promoting embryonic development, wound healing, as well as nerve and lung regeneration. Some researchers reported that $\mathrm{P} 311$ transfection into fibroblast cells induced phenotypic changes consistent with myofibroblast transformation, decreased TGF- $\beta 1$ signaling and caused an inhibition in collagen expression (15). Their findings suggested that P311 may be involved in facilitating wound healing and/ or minimizing scarring during wound repair via preventing fibrosis.

Although previous study has suggested P311 may be an important factor in myofibroblast transformation and in the progression of fibrosis, the related studies on P311 on renal fibrosis are limited and the mechanisms of P311 in the progression of renal tubulointerstitial fibrosis remain largely unknown. In our previous studies, we found that P311 may be involved in the pathogenesis of renal fibrosis by inhibiting EMT process via TGF- $\beta 1-$ Smad-ILK pathway in NRK-52E cells (20). In the present study, we further examined the effect of P311 on TGF- $\beta 1$-mediated EMT in a rat model of unilateral ureteral occlusion (UUO) renal fibrosis. After construction the recombinant adenovirus p311 (also called Ad-P311) was transferred it into UUO rats, the preventing effect and possible mechanism of P311 on TGF- $\beta 1$-mediated EMT were explored.

\section{Materials and methods}

Animals. Forty male Sprague-Dawley rats (8-week-old, $200 \pm 10 \mathrm{~g}$ ) were purchased from Shandong Experimental Animal Center (Jinan, China), and were given free access to water and food throughout the experiments. The rats were acclimatized for at least 1 week prior to the experiments. All of the animal experimental protocols were handled in accordance with the Code of Ethics of the World Medical Association, and all research procedures were permitted by Medical Ethics Committee of Provincial Hospital Affiliated to Shandong University.
Establishment of the UUO model. UUO was performed as described previously (21). Briefly, after induction of general anesthesia by intraperitoneal injection of $3 \%$ pentobarbital (Sigma, St. Louis, MO, USA) (1 ml/kg body wt), the abdominal cavity was exposed via midline incision and the left ureter was ligated at 2 points with 4-0 silk (Niccho Kogyo Co., Ltd., Tokyo, Japan). Ureters of the sham-operated rats were manipulated, but not ligated, and were used as controls.

Experimental protocol. The rats were randomly divided into four groups and given different treatment, each group consisted of ten animals as follows: control group (or sham surgery group), UUO group, control + Ad-P311 group, and UUO + Ad-P311 group. Ad-P311 was constructed as described previously by the current authors and stored at $-80^{\circ} \mathrm{C}$ for use (20). After establishment of the UUO model successfully, the rats in control group and UUO group were injected with $0.5 \mathrm{ml}$ normal saline through the tail vein each week for 4 weeks, while the rats in control + Ad-P311 group and UUO + Ad-P311 group were injected with $0.5 \mathrm{ml} \mathrm{P} 311$ adenovirus by tail vein per week for 4 weeks. Then, rats were sacrificed and their kidneys were taken and blood analysis was performed. Serum creatinine $(\mathrm{Cr})$, blood urea nitrogen (BUN) and albumin (ALB) (BioVision, Inc., Milpitas, CA, USA) levels in the blood were tested according to the introduction of detection kits. Part of the kidneys was fixed in $10 \%$ formalin solution and embedded in paraffin as $3 \mu \mathrm{m}$ sections for hematoxylin and eosin (H\&E), Masson's trichrome and immunohistochemical staining. The other part of the kidneys was stored at $-80^{\circ} \mathrm{C}$ for western blot analysis, which was performed as previously described to detect the protein expressions of E-cadherin, $\alpha$-SMA, TGF- $\beta 1$, phosphorylated Smad2/3 (pSmad2/3), Smad7 and ILK (Santa Cruz Biotechnology, Inc., Santa Cruz, CA, USA).

$H \& E$ staining and Masson staining. To observe the histological morphology changes of kidney tissue, H\&E staining and Masson staining were performed. First, kidney tissues were fixed with $10 \%$ neutral formaldehyde and dehydrated in graded ethanol. After permeation in xylene, they were embedded in paraffin. The paraffin blocks were cut into $2 \mu \mathrm{m}$ slices, mounted onto glass slides and stained by standard techniques of H\&E staining and Masson staining according to previous studies $(10,22)$. Renal tubular injury index including inflammation, cell infiltration, interstitial fibrosis, interstitial edema, cell vacuolar degeneration, tubular atrophy, and tubular expansion were measured to assess the renal interstitial lesions. Ten different fields were selected to estimate the level of renal injury index with H\&E staining using bio-image analysis system (Bio-Profile). Each parameter was evaluated and given a score from 0 to $4^{+},\left(0\right.$, no changes; $1^{+}$, changes affecting $5-25 \%$ of the sample; $2^{+}$, changes affecting $25-50 \% ; 3^{+}$, changes affecting $50-75 \% ; 4^{+}$, changes affecting $\left.75-100 \%\right)(23)$. The severity of interstitial fibrosis was estimated by scanning 10 no-repeated fields in each sample with Masson staining. Blue-stained fibrotic areas were quantified by the Image-Pro plus 6.0 software (Media Cybernetics, Rockville, MD, USA) (23). The results were expressed by the proportion of the relative volume of the scanned interstitium. The cases with H\&E and Masson staining were evaluated by two investigators independently and any discrepancy was resolved by a group discussion. 


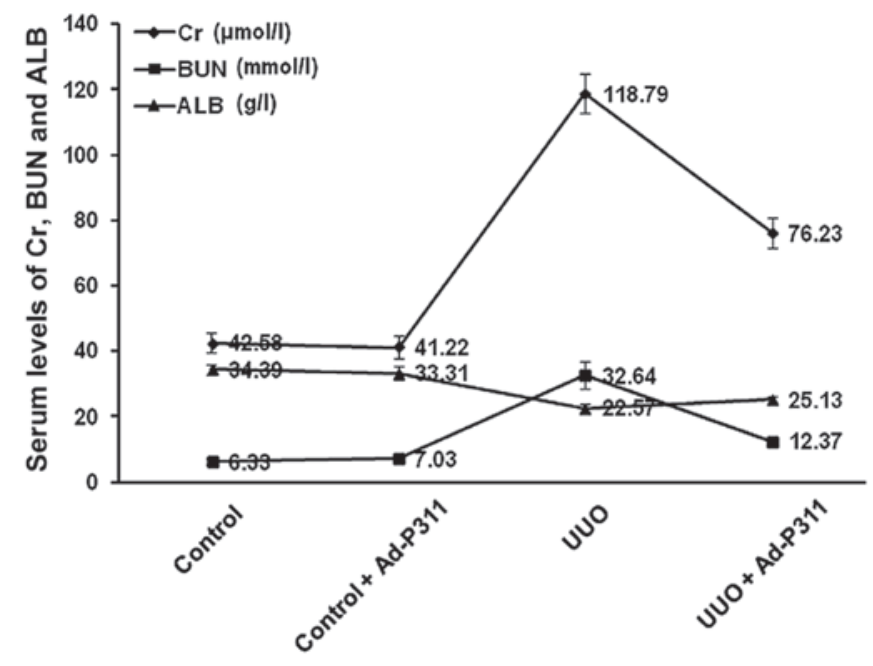

Figure 1. The serum levels of $\mathrm{Cr}$, blood urea nitrogen (BUN) and albumin (ALB) in unilateral ureteral occlusion (UUO) rats and Ad-P311 administered UUO rats.

Immunohistochemical staining. To analyze the protein expression of $\alpha$-SMA, pSmad2/3 and Smad7, immunohistochemical staining was performed as described previously (22). First, paraffin sections were de-paraffinized, hydrated, and immersed in $0.3 \%$ hydrogen peroxide in methanol for 30 min to block the endogenous peroxidase activity. Second, the sections were incubated in primary antibodies $(\alpha-S M A$, Smad $2 / 3$ and Smad7) overnight at $4^{\circ} \mathrm{C}$, followed by incubation in anti-mouse secondary antibody for $1 \mathrm{~h}$ at room temperature. Third, visualization was carried out using the DAB horseradish peroxidase color development kit (Beyotime Institute of Biotechnology, Shanghai, China), and slides were counter-stained in hematoxylin-1. Finally, ten random fields were examined per slice for expression of $\alpha$-SMA, Smad2/3, and Smad7 using a Leica DM2500 optical microscope at a magnification of $x 200$. The mean optical density (MOD) was measured by Image-Pro Plus 6.0 image analysis software. All cases in immunohistochemical staining were evaluated by two investigators independently and any discrepancy was resolved by a group discussion.

Western blot analysis. Thirty micrograms of total cellular proteins were resolved by sodium dodecyl sulfate-polyacrylamide gel electrophoresis (SDS-PAGE) and transferred onto polyvinylidene fluoride (PVDF) transfer membranes by western blotting (24). The results were quantified using ImageJ (National Institutes of Health, Bethesda, MD, USA). The following antibodies were used: E-cadherin, $\alpha$-SMA, TGF- $\beta 1, \mathrm{pSmad} 2 / 3, \operatorname{Smad} 7$ and ILK (Santa Cruz Biotechnology, Inc.).

Statistical analysis. Statistical analysis was performed using SPSS software, version 17.0 (SPSS, Inc., Chicago, IL, USA). All experiments were performed in triplicate and the data are expressed as the mean \pm standard deviation (SD). The statistical significance of differences was calculated using the t-test and one-way analysis of variance (ANOVA), and $\mathrm{p}<0.05$ was considered statistically significant.

\section{Results}

The general condition of rats. After treatment for 4 weeks, the rats in control group and control + Ad-P311 group were still in good shape, glossy coat color and obesity, while the rats in UUO model group had different degrees of anorexia, low spirits, matt coat color, and kidney enlargement, and even individual rats were dead from kidney failure (there were 5 deaths including 3 in UUO group and 2 in UUO + Ad-P311 group). However, the above symptoms were improved by the administration of Ad-P311 in UUO rats. In addition, there was a great change in body weight of rats after establishment of the UUO model and treatment with Ad-P311 (data not shown). After 4 weeks of treatment, body weights in UUO group $(\mathrm{p}<0.01)$ and UUO + Ad-P311 group $(\mathrm{p}<0.05)$ had different degrees of weight loss compared to the control group and control + Ad-P311 group, while there was no significant difference in control group and control + Ad-P311 group ( $p>0.05$ ). Moreover, the weight loss was reversed significantly by the administration of Ad-P311 in UUO + Ad-P311 group compared to that in UUO group $(\mathrm{p}<0.05)$.

The serum levels of $C r, B U N$ and $A L B$. As shown in Fig. 1, the serum levels of $\mathrm{Cr}$ and BUN in UUO group (Cr 118.79 $\pm 6.14 \mu \mathrm{mol} / \mathrm{l}$; BUN 32.64 $\pm 4.02 \mathrm{mmol} / \mathrm{l}$ ) were higher than those in control group $(\mathrm{Cr} 42.58 \pm 2.95 \mu \mathrm{mol} / \mathrm{l}$; BUN 6.33 $\pm 1.1 \mathrm{mmol} / \mathrm{l})$ and control + Ad-P311 group $(\mathrm{Cr}$ $41.22 \pm 3.36 \mu \mathrm{mol} / 1$; BUN $7.03 \pm 0.90 \mathrm{mmol} / \mathrm{l})(\mathrm{p}<0.01)$, while there was no significant difference in control group and control + Ad-P311 group ( $\mathrm{p}>0.05)$. However, Ad-P311 administration substantially decreased the serum levels of $\mathrm{Cr}$ and BUN. The serum levels of $\mathrm{Cr}$ and BUN in UUO + Ad-P311 group (Cr 76.23 $\pm 4.45 \mu \mathrm{mol} / \mathrm{l}$; BUN $12.37 \pm 1.33 \mathrm{mmol} / \mathrm{l}$ ) were lower than those in UUO group $(\mathrm{p}<0.01)$. The serum levels of ALB in UUO group (ALB 22.57 $\pm 1.43 \mathrm{~g} / \mathrm{l}$ ) were higher than those in control group (ALB 34.39 $\pm 1.66 \mathrm{~g} / \mathrm{l}$ ) and control + Ad-P311 group (ALB 33.31 $\pm 1.95 \mathrm{~g} / \mathrm{l})(\mathrm{p}<0.01)$, while there was no significant difference in control group and control + Ad-P311 group ( $\mathrm{p}>0.05)$. However, Ad-P311 administration substantially increased the serum levels of ALB. The serum levels of ALB in UUO + Ad-P311 group (ALB 25.13 $\pm 0.97 \mathrm{~g} / \mathrm{l}$ ) were higher than those in UUO group $(\mathrm{p}<0.01)$.

Ad-P311 attenuated UUO-induced fibrosis. H\&E staining demonstrated that there were no histological changes in the kidneys of sham rats as shown in control group (Fig. 2A-a) and control + Ad-P311 group (Fig. 2A-b). By contrast, the kidneys developed remarkable pathological changes such as interstitial fibrosis, tubular expansion, and atrophy and inflammatory cell invasion in UUO rats as shown in UUO group (Fig. 2A-c). However, these histological lesions in the kidneys of UUO rats were attenuated by the administration of Ad-P311 as shown in UUO + Ad-P311 group (Fig. 2A-d). Consistent with the pathological changes in the experimental kidneys, UUO surgery resulted in $\sim 8$-fold increase of the renal tubular injury index, but the extent of damage was remarkably decreased from $83.20 \pm 2.80 \%$ (UUO group) to $75.60 \pm 3.40 \%$ (UUO + Ad-P311 group) by the administration of Ad-P311 (Fig. 2B). Masson staining showed that collagen deposition and fibrosis area were significantly decreased in UUO + Ad-P311 group 


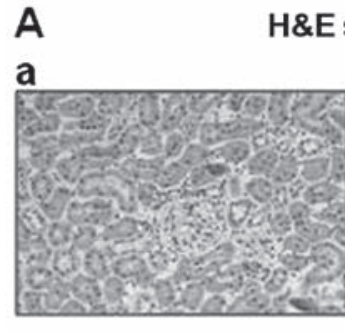

Control

c

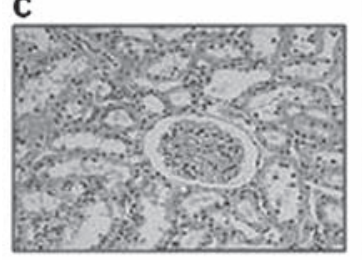

UUO
H\&E staining

b

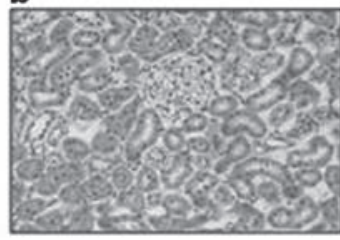

Control+ Ad-P311

d

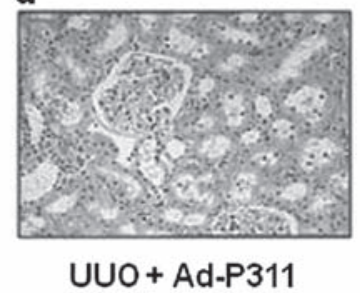

B

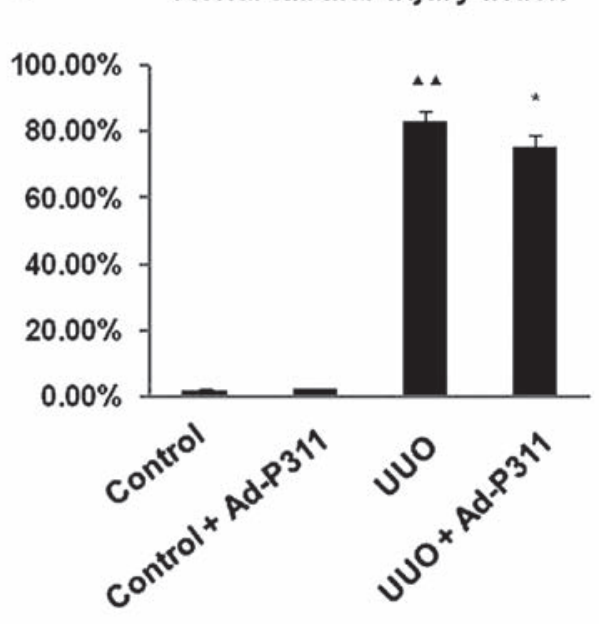

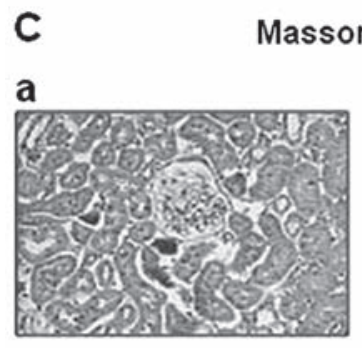

Control

c

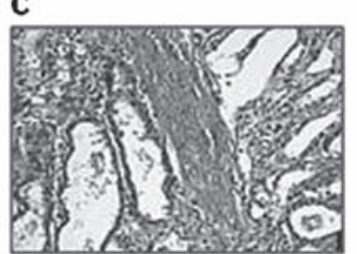

UUO b

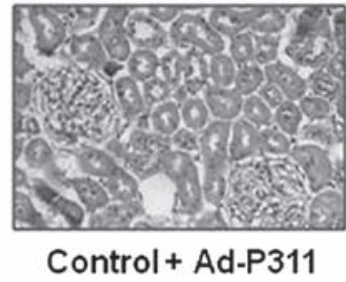

d

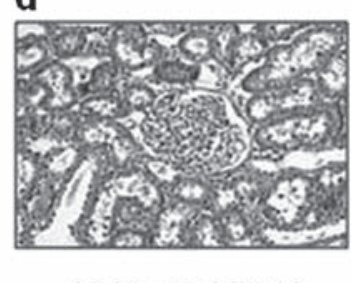

D Collagen disposition area

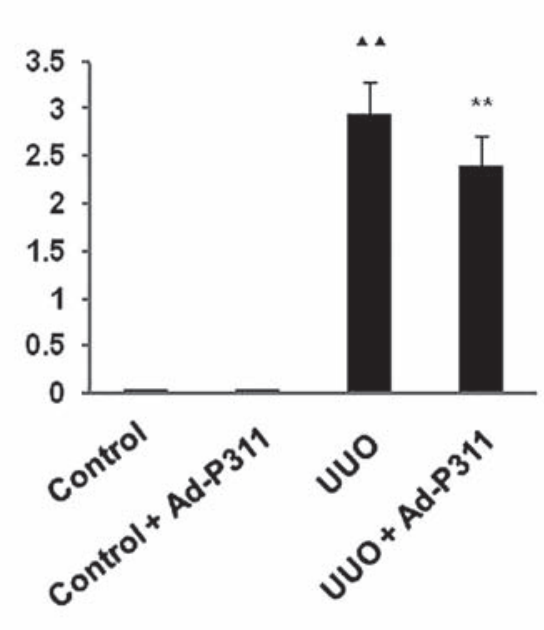

Figure 2. Ad-P311 attenuates unilateral ureteral occlusion (UUO)-induced pathological changes in the kidneys. (A) H\&E staining (x200). (B) The semiquantitative accession of the renal tubular injury index in H\&E-stained sections of rat kidneys (C) Masson staining (x200). (D) The degree of interstitial collagen deposits in Masson-stained sections of rat kidneys.

compared to UUO group (Fig. 2C). The collagen accumulation had prominently increased $\sim 3$-fold in the renal interstitium of UUO group, while administration of Ad-P311 decreased the amount of collagen deposition from $2.94 \pm 0.34$ (UUO group) to $2.39 \pm 0.33$ (UUO + Ad-P311 group), compared to UUO group (p<0.01) (Fig. 2D).

Ad-P311 reverses the expression $\alpha-S M A, p S m a d 2 / 3$ and Smad7 detected by immunohistochemical staining in UUO kidneys. To explore the effect of Ad-P311 on EMT-related markers in UUO kidneys, the expression of the mesenchymal marker $\alpha$-SMA in UUO kidneys was investigated by immunohistochemical staining. As shown in Fig. 3A and B, the protein expression of $\alpha$-SMA was increased significantly in UUO group compared to that in the sham operated control kidneys (control group and control + Ad-P311 group). In contrast, the increased expression of $\alpha$-SMA in UUO group was reversed by the administration of Ad-P311 as shown in UUO + Ad-P311 group.
Furthermore, to explore the possible mechanism of Ad-P311 on EMT of UUO kidneys, the protein expression of Smad2/3 and Smad7 was investigated by immunohistochemical staining. As shown in Fig. $3 \mathrm{C}$ and D, the protein expression of $\mathrm{pSmad} 2 / 3$ was dramatically elevated in UUO group compared with the sham groups (control group and control + Ad-P311 group), but Ad-P311 administration (UUO + Ad-P311 group) substantially ameliorated this elevation induced by UUO surgery (UUO group). As shown in Fig. 3E and F, the protein expression of $\mathrm{Smad} 7$ was decreased significantly in UUO group compared to that in the sham groups (control group and control + Ad-P311 group), while the administration of Ad-P311 alleviated its decrease as shown in UUO + Ad-P311 group.

Ad-P311 reversed the expression of EMT related proteins detected by western blot analysis in UUO kidneys. To further explore the effect of Ad-P311 on EMT related markers in UUO kidneys, the expression of the epithelial marker E-cadherin, 

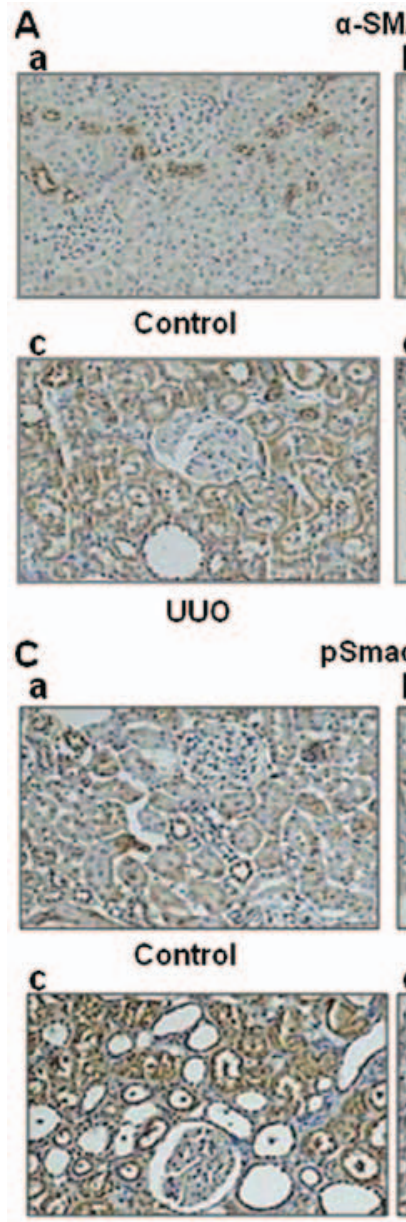

UUO

E
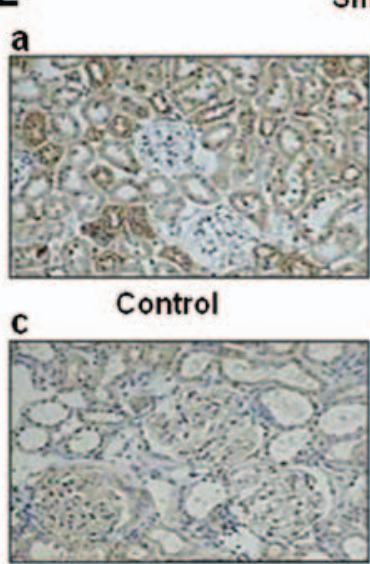

UUO
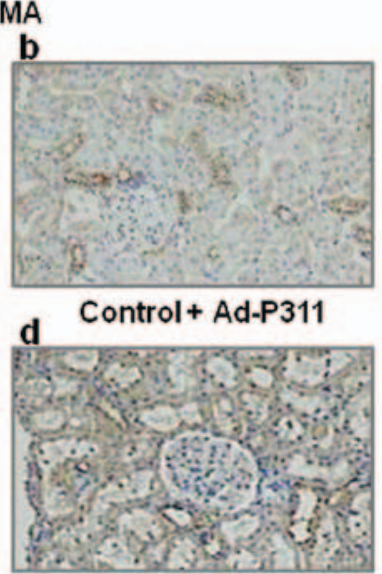

UUO + Ad-P311

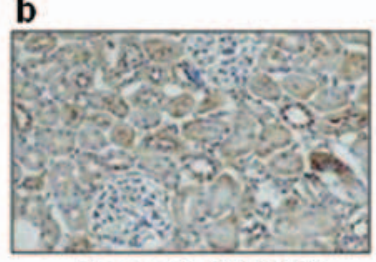

Control+ Ad-P311

d

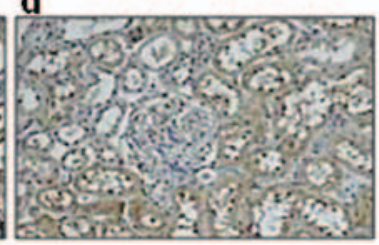

UUO + Ad-P311

Smad7

b

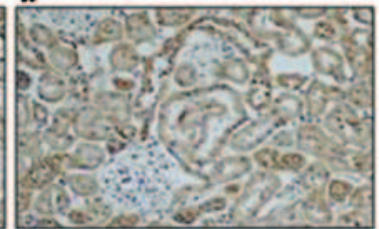

d Control+Ad-P311

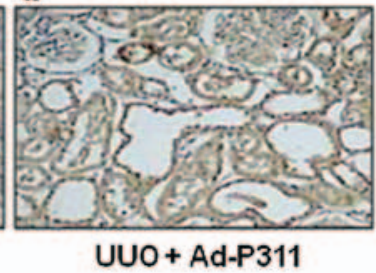

B

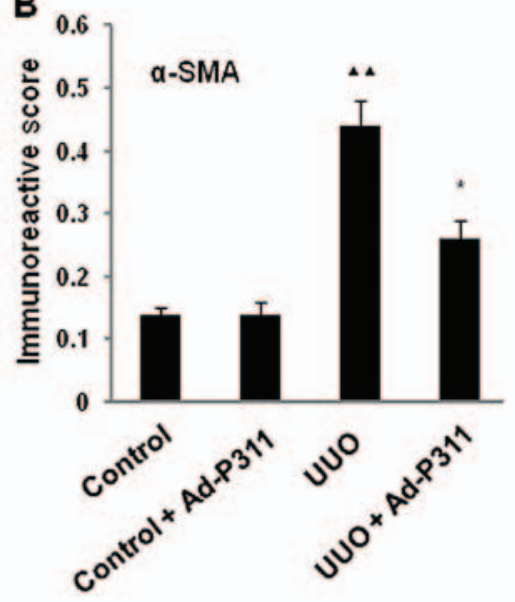

D

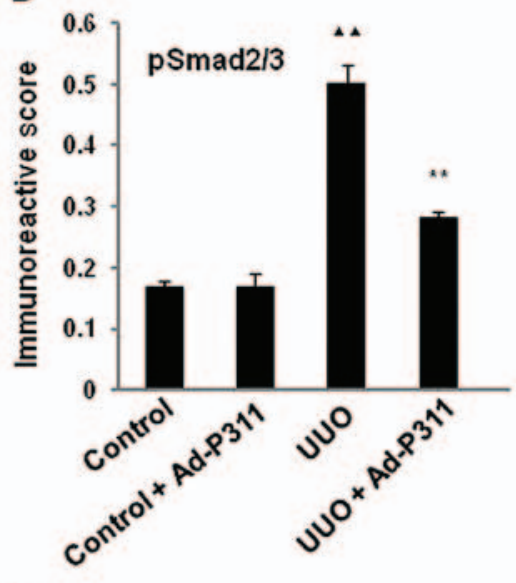

$\mathbf{F}$

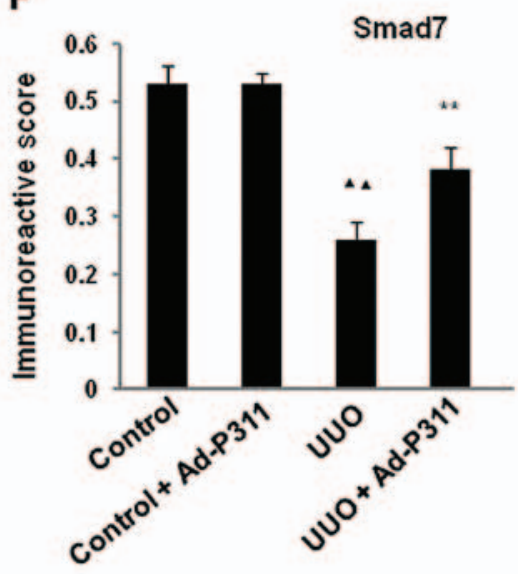

Figure 3. Ad-P311 reverses the expression $\alpha$-smooth muscle actin $(\alpha$-SMA), pSmad2/3 and Smad7 detected by immunohistochemical staining in unilateral ureteral occlusion (UUO) kidneys. (A) Representative photomicrograph illustrating the $\alpha$-SMA expression in UUO kidneys. (B) Graph showing the immunoreactive score of $\alpha$-SMA in UUO kidneys by quantitative morphometric analysis. (C) Representative photomicrograph illustrating the pSmad2/3 expression in UUO kidneys. (D) Graph showing the immunoreactive score of pSmad2/3 in UUO kidneys by quantitative morphometric analysis. (E) Representative photomicrograph illustrating the Smad7 expression in UUO kidneys. (F) Graph showing the immunoreactive score of Smad7 in UUO kidneys by quantitative morphometric analysis. ${ }^{\wedge} \mathrm{p}<0.01$ compared to the control group; ${ }^{*} \mathrm{p}<0.05$ compared to the UUO group; ${ }^{* *} \mathrm{p}<0.01$ compared to the UUO group. Original magnifications, $\times 200$.

and the mesenchymal marker $\alpha$-SMA in UUO kidneys was examined by western blot analysis. As shown in Fig. 4, the protein level of E-cadherin was decreased significantly in UUO group compared with the sham groups (control group and control + Ad-P311 group), but administration of Ad-P311 alleviated its decrease as shown in UUO + Ad-P311 group.
In contrast, the protein expression of $\alpha$-SMA was increased significantly in UUO group compared to that in the sham operated control kidneys (control group and control + Ad-P311 group), while the increased expression of $\alpha$-SMA in UUO group was reversed by the administration of Ad-P311 as shown in UUO + Ad-P311 group (Fig. 4). 

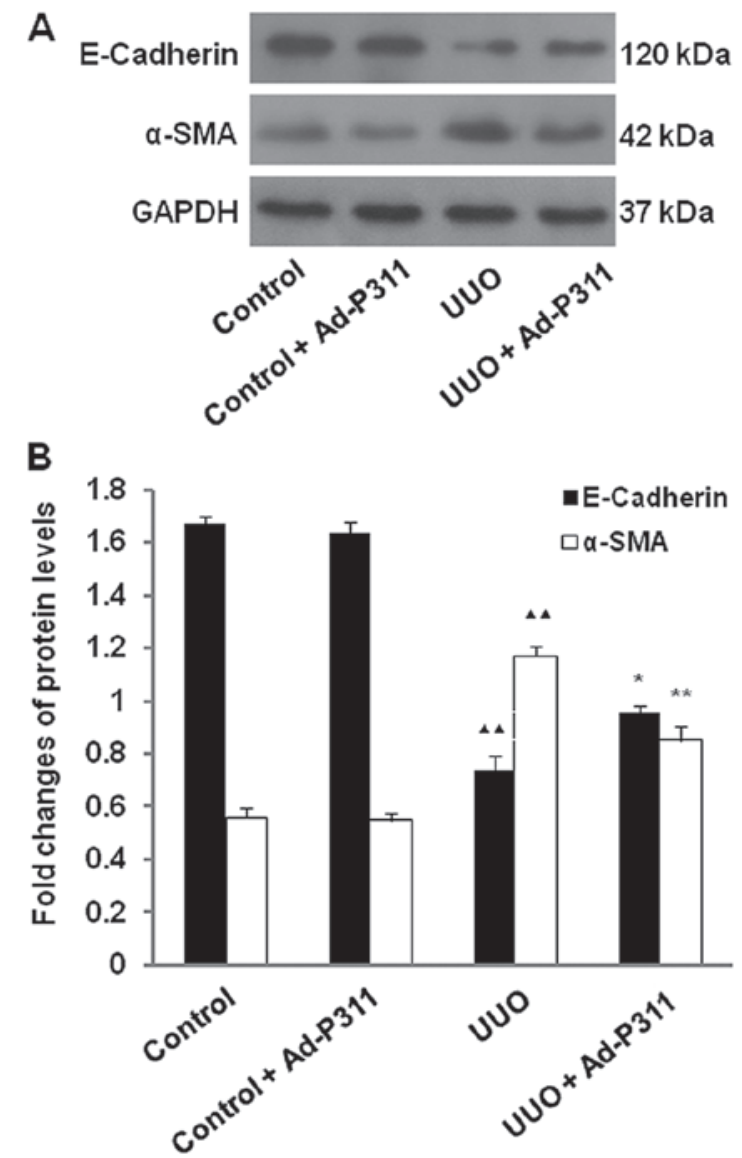

Figure 4. Ad-P311 reverses the expression of epithelial-mesenchymal transition (EMT) markers [E-cadherin and $\alpha$-smooth muscle actin ( $\alpha$-SMA) detected by western blot analysis in unilateral ureteral occlusion (UUO) kidneys]. (A) Expression of E-cadherin and $\alpha$-SMA at the protein level in each group were determined with GAPDH used as an internal control. (B) The expression level of E-cadherin and $\alpha$-SMA was quantitatively analyzed with ImageJ software. ${ }^{\wedge} \mathrm{p}<0.01$ compared to the control group; ${ }^{*} \mathrm{p}<0.05$ compared to the UUO group; ${ }^{* *} \mathrm{p}<0.01$ compared to the UUO group.

To explore the possible mechanism of Ad-P311 on EMT of UUO kidneys, the protein expression of EMT-related proteins TGF- $\beta 1$, pSmad 2/3, Smad7 and ILK were measured by western blot analysis. As shown in Fig. 5, the protein expression of TGF- $\beta 1, \mathrm{pSmad} 2 / 3$ and ILK were increased significantly in UUO group compared to that in the sham operated control kidneys (control group and control + Ad-P311 group), but administration of Ad-P311 alleviated all of above changes significantly as shown in UUO + Ad-P311 group. In contrast, the protein expression of Smad7 was decreased significantly in UUO group compared to that in the sham operated control kidneys (control group and control + Ad-P311 group), while the decreased expression of Smad7 in UUO group was reversed by the administration of Ad-P311 as shown in UUO + Ad-P311 group (Fig. 5).

\section{Discussion}

P311 is a conserved $8-\mathrm{kDa}$ intracellular protein expressed in vascular and visceral smooth muscle beds, the nervous system, regenerating tissues as well as malignant glioblastomas $(16,17,19,25)$. Previous studies have suggested P311 may be an important factor in myofibroblast transformation and in
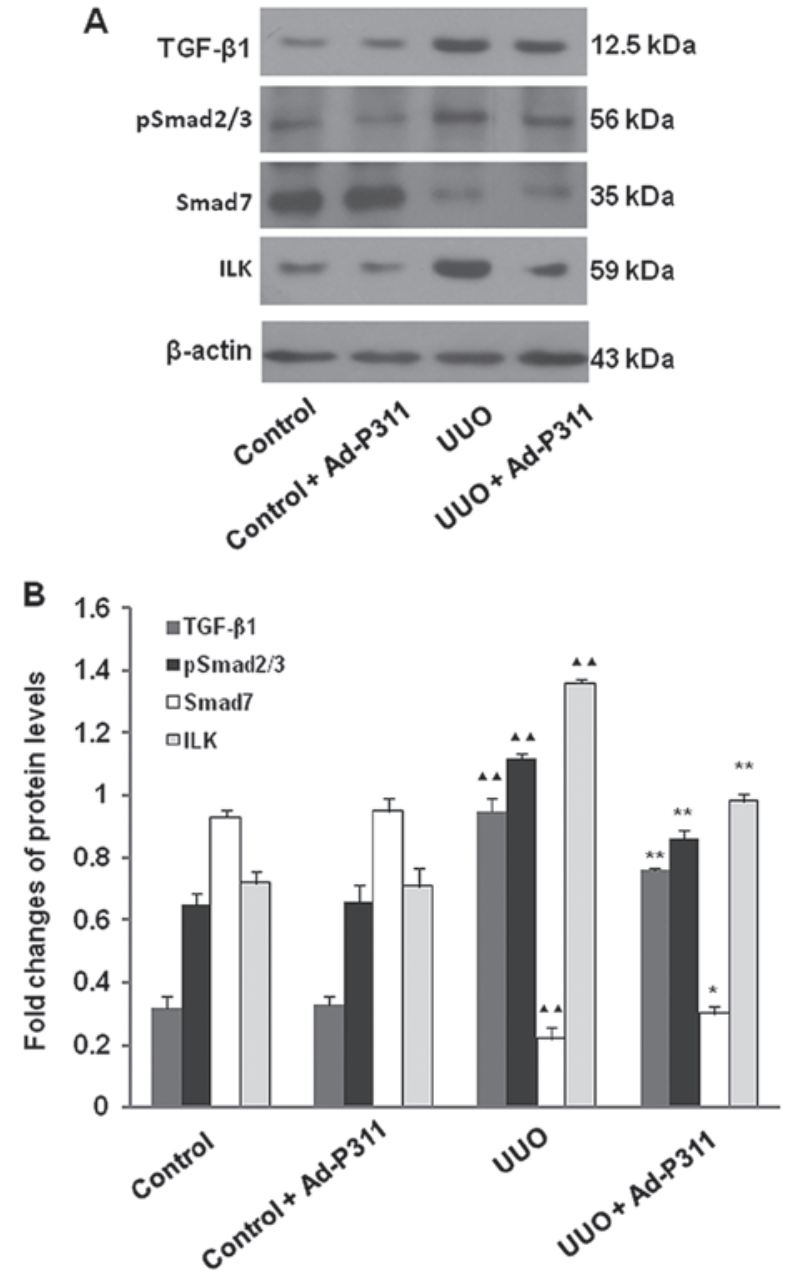

Figure 5. Ad-P311 reverses the expression of epithelial-mesenchymal transition (EMT)-related proteins transforming growth factor- $\beta 1$ (TGF- $\beta 1$ ), pSmad2/3, Smad7 and integrin linked kinase (ILK) detected by western blot analysis in unilateral ureteral occlusion (UUO) kidneys. (A) Expression of TGF- $\beta 1, \mathrm{pSmad} 2 / 3$, Smad7 and ILK at the protein level in each group was determined with $\beta$-actin used as an internal control. (B) The expression level TGF- $\beta 1$, pSmad $2 / 3$, Smad7 and ILK was quantitatively analyzed with ImageJ software. ${ }^{\wedge} \mathrm{p}<0.01$ compared to the control group; ${ }^{*} \mathrm{p}<0.05$ compared to the UUO group; ${ }^{* *} \mathrm{p}<0.01$ compared to the UUO group.

the progression of fibrosis (13-20). However, the related studies on P311 on renal fibrosis are limited and the mechanisms of P311 in the progression of CKD remain largely unknown. In our previous studies, we found that P311 may be involved in the pathogenesis of renal fibrosis by inhibiting EMT process via TGF- $\beta 1$-Smad-ILK pathway in NRK-52E cells (20). In our previous studies, we also determined whether p311 expressed in the kidney of rats transfected with Ad-p311 by quantitative real-time RT-PCR assay. P311 was expressed in the kidneys of UUO rats but not in the kidneys of sham surgery rats. Furthermore, P311 gene could be highly and stably transfected into UUO rats with adenovirus mediation (data not shown). Thus, in the present study, the effect and possible mechanism of adenovirus-mediated P311 on TGF- $\beta 1$-mediated EMT in UUO rats were further investigated. We found that adenovirus-mediated P311 was capable of improving fibrosis by regulating expressions of EMT related markers in vivo and the underlying mechanisms may involve the TGF- $\beta 1$-Smad-ILK signaling pathway. 


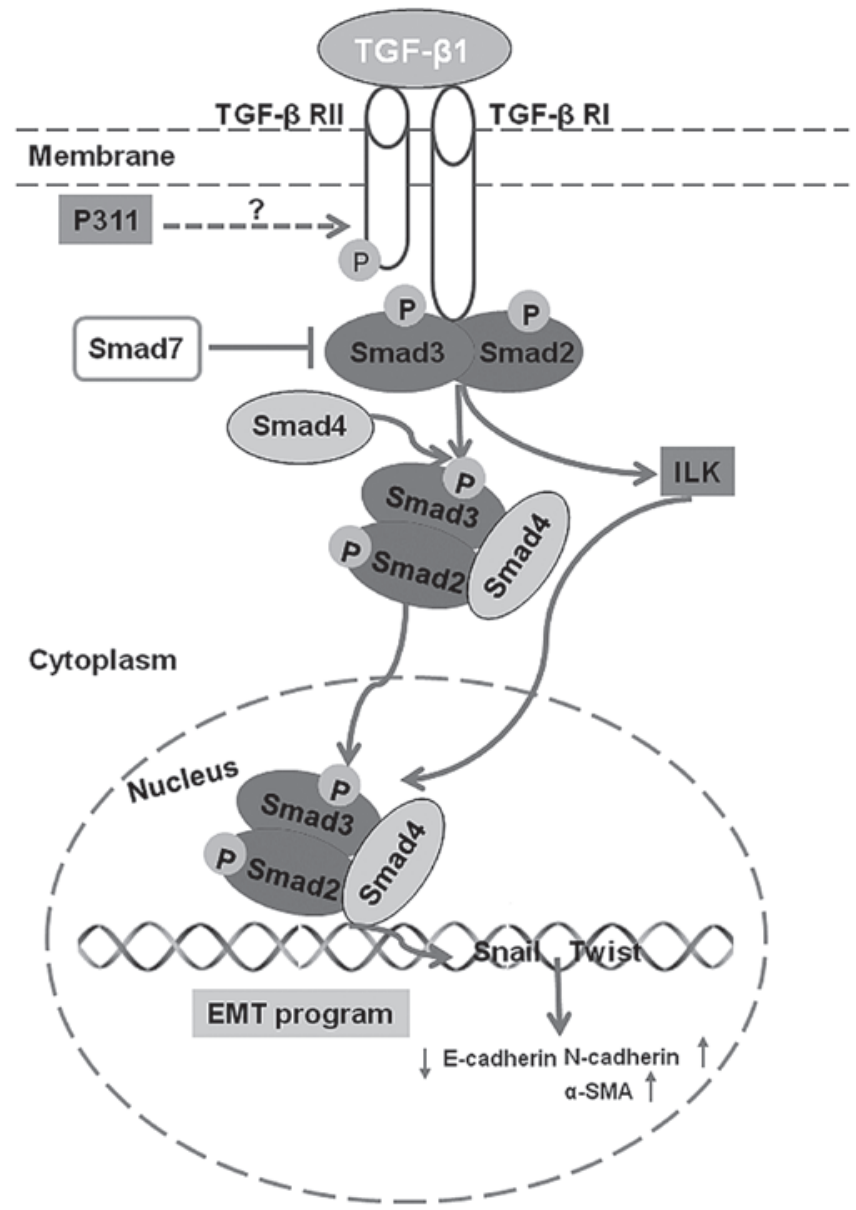

Figure 6. The possible mechanisms of P311 involved in the transforming growth factor- $\beta 1$ (TGF- $\beta 1$ )-mediated epithelial-mesenchymal transition (EMT) in renal fibrosis via TGF- $\beta 1$-Smad-integrin linked kinase (ILK) pathway.

CKD is characterized by an irreversible decrease in kidney function and renal fibrosis is considered as a common pathological hallmark of progressive CKD (26). UUO is recognized as an ideal experimental model of renal interstitial fibrosis. Ureteral obstruction leads to a significant inflammatory and fibrotic response, followed by infiltration of inflammatory cells, renal interstitial myofibroblast proliferation, and ECM accumulation in the renal interstitium (27). We established the UUO model successfully and administered Ad-P311 into UUO rats by the tail vein. Then the general condition of rats and the serum levels of $\mathrm{Cr}, \mathrm{BUN}$ and ALB were evaluated. The rats in UUO model group had different degrees of anorexia, low spirits, matt coat color, kidney enlargement, weight loss, and even individual rats dead from kidney failure, but these symptoms were improved by the administration of Ad-P311 in UUO rats. Moreover, the serum levels of $\mathrm{Cr}$ and BUN were increased significantly and the serum levels of ALB were decreased significantly in the UUO rats. However, the increased serum levels of $\mathrm{Cr}$ and BUN and the decreased serum levels of ALB were reversed by the administration of Ad-P311 in UUO rats. In addition, H\&E staining and Masson staining indicated that the kidneys developed remarkable pathological changes such as interstitial fibrosis, tubular expansion, and atrophy and inflammatory cell invasion in UUO rats. However, these histological lesions in the kidneys of UUO rats were attenuated after administration of Ad-P311 with the renal tubular injury index and collagen disposition area reversed. These findings suggested Ad-P311 alleviated kidney function and renal fibrosis in UUO rats.

Renal fibrosis is generally considered a failure of tissue injury/repair response, which is closely associated with chronic interstitial inflammation (11). The progression of renal fibrosis primarily involves transdifferentiation of renal fibroblasts into myofibroblasts and infiltration of inflammatory cells, with production and release of profibrotic cytokines and growth factors, such as TGF- $\beta 1$, TNF- $\alpha$, IL- $1 \beta$, IL- 6 , and PDGF (28). TGF- $\beta 1$ as a key paracrine/autocrine growth factor and profibrotic cytokine plays a key role in renal fibrosis, which promotes tubular epithelial myofibroblast transdifferentiation and increases the synthesis and accumulation of ECM proteins $(29,30)$. P311 is an intracytoplasmic protein that can bind to TGF- $\beta$-latent associated protein (LAP) and downregulates the expression of TGF- $\beta 1$ and TGF- $\beta 2$ (14). Moreover, Pan et al have reported that P311 in myofibroblast transformation could decrease TGF- $\beta 1$ signaling and cause inhibition in collagen expression, suggesting that P311 may be involved in preventing fibrosis during wound repair (15).

In the present study, the protein expression of TGF- $\beta 1$ was increased significantly in UUO kidneys, but administration of Ad-P311 alleviated its increase. These findings indicated that adenovirus-mediated P311 could ameliorate renal fibrosis through regulating TGF- $\beta 1$ in UUO rats. However, Yao et al used $\mathrm{P} 311^{-/}$and $\mathrm{P} 311^{+/+} \mathrm{C} 57 \mathrm{BL} / 6$ mice to establish UUO model and found that $\mathrm{P} 311$ could promote renal fibrosis via TGF $\beta 1 /$ Smad signaling (31). This finding differs from our data. Although the previous study suggested P311 may be an important factor in myofibroblast transformation and in the progression of fibrosis, the related studies of P311 on renal fibrosis are limited and the mechanisms of P311 in the progression of renal fibrosis remains largely unknown. Thus, it is difficult to explain this discrepancy with our results. The authors consider the following reasons may be responsible for the discrepancy. Since P311 inhibited profibrotic cytokine TGF- $\beta 1$ accompanied with the resultant decrease in collagen expression, P311 levels may be one of the factors contributing to determine whether a lesion will heal faster or with less fibrosis (15). It should be stressed, however, that although P311 inhibits TGF- $\beta 1$ synthesis, myofibroblasts remain responsive to exogenous TGF- $\beta 1$, as the inhibition effect of P311 on collagen was overcome by exogenous TGF- $\beta 1$. Moreover, noteworthy that myofibroblasts are not the only source of TGF- $\beta 1$, which is mainly produced by inflammatory cells normally present at the wound site (32-34). Therefore, depending on the magnitude and duration of the inflammatory response, the autocrine antifibrogenic effect of P311 may be partially counterbalanced or completely offset by the paracrine production of TGF- $\beta 1$ (15). In a noncomplicated wound, however, inflammatory cells are present at early stages of repair, and then gradually disappear from the site. Thus, the antifibrogenic effect of P311 may increase over time and become maximal toward the end of the reparative process (15). This seems interesting as the relationship between P311 and TGF- $\beta 1$ is like two competitors sitting on a seesaw. During renal fibrosis, at early stages of repair with abundant inflammatory cells, the antifibrogenic effect of P311 may be offset by TGF- $\beta 1$ and exhibit pro-fibrogenic effect, 
while at advanced stages of repair with inflammatory cells gradually disappeared, the antifibrogenic effect of P311 may increase over time and become maximal toward the end of the reparative process.

Tubular EMT has been widely accepted as the underlying mechanisms of renal fibrosis (26). Damaged tubular epithelial cells undergo EMT and then transfer crucial signals towards renal interstitial to activate phenotypic transition of fibroblasts and sustain inflammation, resulting in progressive renal fibrosis (35). In CKD, TGF- $\beta 1$ is considered a crucial regulator of EMT which mediates the initiation and progression of interstitial fibrosis (31). It can induce the activation of fibroblasts to undergo a phenotypic transition to myofibroblasts with tubular epithelial cells losing their adhesion molecules (such as E-cadherin), and gaining the mesenchymal cell markers (such as $\alpha$-SMA) (4). In the present study, the protein expression of $\alpha$-SMA was increased significantly and the protein expression of E-cadherin was decreased significantly in UUO kidneys compared to that in the sham operated control kidneys, while the changed expressions of $\alpha$-SMA and E-cadherin in UUO kidneys was reversed by the administration of Ad-P311. These results indicated that $\mathrm{P} 311$ could prevent TGF- $\beta 1$-mediated EMT in UUO kidneys.

The TGF- $\beta 1 /$ Smad signaling pathway has been reported to play a key role in initiating and completing the entire EMT course in fibrotic disease pathogenesis (36). During this signaling pathway, both Smad2 and Smad3 proteins are phosphorylated in response to TGF- $\beta$ receptor activation and in subsequent events become downstream mediators of TGF- $\beta$ signaling. Phosphorylated Smad2 and Smad3 then bind to the common Smad4 and form the Smad complex, which translocates into the nucleus to regulate the target gene transcription, including Smad7 (32). Here we found that $\mathrm{pSmad} 2 / 3$ expression was increased and Smad7 expression was decreased significantly in UUO kidneys compared to that in the sham operated control kidneys, but administration of Ad-P311 alleviated all of above changes significantly. These results indicated that P311 regulates not only TGF- $\beta 1$ but also the Smad signaling pathway, which may be involved in the regulation of renal fibrosis by P311. ILK is a serine/threonine protein kinase and a major regulator of integrin signaling, interacting with the cytoplasmic domains of integrin $\beta 1$ and $\beta 3$ subunits. It has a fundamental role in the regulation of cell survival, proliferation, and migration (37). TGF- $\beta 1$-mediated EMT has been suggested to be dependent on ILK function during renal fibrosis depending on intracellular Smad signaling (11). Therefore, ILK is shown to be a key intracellular mediator that controls TGF- $\beta 1$-induced-EMT in renal tubular epithelial cells. We studied the effects of P311 on ILK expression, which was the important downstream mediator of TGF- $\beta 1 /$ Smads signaling pathway. Here we found that ILK expression was increased significantly in UUO kidneys compared to that in the sham operated control kidneys, however, administration of Ad-P311 significantly reversed of above changes. Taken together, these findings indicated that P311 attenuated TGF- $\beta 1$-mediated EMT via regulating the protein expression of $\mathrm{pSmad} 2 / 3$, Smad7 and ILK in UUO kidneys.

In conclusion, our data suggest that P311 may be involved in the pathogenesis of renal fibrosis by blocking TGF- $\beta 1$-mediated EMT via TGF- $\beta 1$-Smad-ILK pathway (Fig. 6) in UUO kidneys.
P311 may be a novel target for the control of renal fibrosis and the progression of CKD.

\section{Acknowledgements}

Not applicable.

\section{Funding}

The present study was supported by the National Natural Science Foundation of China (no. 81273682) and the Science and Technology Development Program of Shandong Province (no. 2010G0020220).

\section{Availability of data and material}

All data generated or analyzed during this study are included in this published article.

\section{Authors' contributions}

GMS was responsible for the concept and design of the study. FHQ, PPC, and XL completed the experiments and performed the data analysis. FHQ was a major contributor in writing the manuscript. All authors read and approved the final manuscript.

\section{Ethics approval and consent to participate}

All the experimental procedures were approved by the Institutional Animal Care and Use Committee of Shandong Provincial Hospital Affiliated to Shandong University (no. 2017-208).

\section{Consent for publication}

Not applicable.

\section{Competing interests}

The authors declare that they have no competing interests.

\section{References}

1. Jha V, Garcia-Garcia G, Iseki K, Li Z, Naicker S, Plattner B, Saran R, Wang AY and Yang CW: Chronic kidney disease: Global dimension and perspectives. Lancet 382: 260-272, 2013.

2. Tonelli M, Wiebe N, Culleton B, House A, Rabbat C, Fok M, McAlister F and Garg AX: Chronic kidney disease and mortality risk: A systematic review. J Am Soc Nephrol 17: 2034-2047, 2006.

3. Boor P, Ostendorf T and Floege J: Renal fibrosis: Novel insights into mechanisms and therapeutic targets. Nat Rev Nephrol 6: 643-656, 2010.

4. Liu Y: New insights into epithelial-mesenchymal transition in kidney fibrosis. J Am Soc Nephrol 21: 212-222, 2010.

5. Kriz W, Kaissling B and Le Hir M: Epithelial-mesenchymal transition (EMT) in kidney fibrosis: Fact or fantasy? J Clin Invest 121: 468-474, 2011.

6. Guo Y, Li Z, Ding R, Li H, Zhang L, Yuan W and Wang Y: Parathyroid hormone induces epithelial-to-mesenchymal transition via the Wnt/ $\beta$-catenin signaling pathway in human renal proximal tubular cells. Int J Clin Exp Pathol 7: 5978-5987, 2014.

7. Wang Q, Wang Y, Huang X, Liang W, Xiong Z and Xiong Z: Integrin $\beta 4$ in EMT: An implication of renal diseases. Int J Clin Exp Med 8: 6967-6976, 2015 
8. Wynn TA: Cellular and molecular mechanisms of fibrosis. J Pathol 214: 199-210, 2008.

9. Impellizzeri D, Esposito E, Attley J and Cuzzocrea S: Targeting inflammation: New therapeutic approaches in chronic kidney disease (CKD). Pharmacol Res 81: 91-102, 2014.

10. Jia L, Ma X, Gui B, Ge H, Wang L, Ou Y, Tian L, Chen Z, Duan Z, Han J, et al: Sorafenib ameliorates renal fibrosis through inhibition of TGF- $\beta$-induced epithelial-mesenchymal transition. PLoS One 10: e0117757, 2015.

11. Kim MK, Maeng YI, Sung WJ, Oh HK, Park JB, Yoon GS, Cho $\mathrm{CH}$ and Park KK: The differential expression of TGF- $\beta 1$, ILK and wnt signaling inducing epithelial to mesenchymal transition in human renal fibrogenesis: An immunohistochemical study. Int J Clin Exp Pathol 6: 1747-1758, 2013

12. Li Y, Yang J, Dai C, Wu C and Liu Y: Role for integrin-linked kinase in mediating tubular epithelial to mesenchymal transition and renal interstitial fibrogenesis. J Clin Invest 112: 503-516, 2003.

13. Penn JW, Grobbelaar AO and Rolfe KJ: The role of the TGF- $\beta$ family in wound healing, burns and scarring: A review. Int $J$ Burns Trauma 2: 18-28, 2012.

14. Paliwal S, Shi J, Dhru U, Zhou Y and Schuger L: P311 binds to the latency associated protein and downregulates the expression of TGF-beta1 and TGF-beta2. Biochem Biophys Res Commun 315: 1104-1109, 2004

15. Pan D, Zhe X, Jakkaraju S, Taylor GA and Schuger L: P311 induces a TGF-beta1-independent, nonfibrogenic myofibroblast phenotype. J Clin Invest 110: 1349-1358, 2002.

16. Mariani L, McDonough WS, Hoelzinger DB, Beaudry C Kaczmarek E, Coons SW, Giese A, Moghaddam M, Seiler RW and Berens ME: Identification and validation of P311 as a glioblastoma invasion gene using laser capture microdissection. Cancer Res 61: 4190-4196, 2001.

17. Fujitani M, Yamagishi S, Che YH, Hata K, Kubo T, Ino H, Tohyama $\mathrm{M}$ and Yamashita T: P311 accelerates nerve regeneration of the axotomized facial nerve. J Neurochem 91: 737-744, 2004.

18. Zhao L, Leung JK, Yamamoto H, Goswami S, Kheradmand F and $\mathrm{Vu} \mathrm{TH}$ : Identification of $\mathrm{P} 311$ as a potential gene regulating alveolar generation. Am J Respir Cell Mol Biol 35: 48-54, 2006.

19. Tan J, Peng X, Luo G, Ma B, Cao C, He W, Yuan S, Li S, Wilkins JA and Wu J: Investigating the role of P311 in the hypertrophic scar. PLoS One 5: e9995, 2010.

20. Qi F, Cai P, Liu X, Peng M and Si G: Adenovirus-mediated P311 inhibits TGF- $\beta 1$-induced epithelial-mesenchymal transition in NRK-52E cells via TGF- $31-S m a d-I L K$ pathway. Biosci Trends 9: 299-306, 2015

21. Baba I, Egi Y, Utsumi H, Kakimoto T and Suzuki K: Inhibitory effects of fasudil on renal interstitial fibrosis induced by unilateral ureteral obstruction. Mol Med Rep 12: 8010-8020, 2015.

22. Yin Y, Qi F, Song Z, Zhang B and Teng J: Ferulic acid combined with astragaloside IV protects against vascular endothelia dysfunction in diabetic rats. Biosci Trends 8: 217-226, 2014.
23. Liu QF, Ye JM, Deng ZY, Yu LX, Sun Q and Li SS: Ameliorating effect of Klotho on endoplasmic reticulum stress and renal fibrosis induced by unilateral ureteral obstruction. Iran J Kidney Dis 9: 291-297, 2015.

24. Lu H, Dong J, Zhang Y, Li C, Yu Q and Tang W: Pathological changes in primary cilia: A novel mechanism of graft cholangiopathy caused by prolonged cold preservation in a rat model of orthotopic liver transplantation. Biosci Trends 8: 206-211, 2014.

25. Badri KR, Yue M, Carretero OA, Aramgam SL, Cao J, Sharkady S, Kim GH, Taylor GA, Byron KL and Schuger L: Blood pressure homeostasis is maintained by a P311-TGF- $\beta$ axis. J Clin Invest 123: 4502-4512, 2013

26. Yoshinaga T, Uwabe K, Naito S, Higashino K, Nakano T, Numata Y and Kihara A: AM251 suppresses epithelial-mesenchymal transition of renal tubular epithelial cells. PLoS One 11: e0167848, 2016.

27. Wang L, Ma J, Guo C, Chen C, Yin Z, Zhang X and Chen X: Danggui buxue tang attenuates tubulointerstitial fibrosis via suppressing NLRP3 inflammasome in a rat model of unilateral ureteral obstruction. Biomed Res Int 2016: 9368483, 2016.

28. Liu Y: Cellular and molecular mechanisms of renal fibrosis. Nat Rev Nephrol 7: 684-696, 2011.

29. Fan JM, Ng YY, Hill PA, Nikolic-Paterson DJ, Mu W, Atkins RC and Lan HY: Transforming growth factor-beta regulates tubular epithelial-myofibroblast transdifferentiation in vitro. Kidney Int 56: 1455-1467, 1999 .

30. Wang F, Xie X, Fan J, Wang L, Guo D, Yang L, Ma X, Zhang L and Li Z: Expression of P311, a transforming growth factor beta latency-associated protein-binding protein, in human kidneys with IgA nephropathy. Int Urol Nephrol 42: 811-819, 2010.

31. Loboda A, Sobczak M, Jozkowicz A and Dulak J: TGF- $\beta 1 /$ Smads and miR-21 in renal fibrosis and inflammation. Mediators Inflamm 2016: 8319283, 2016.

32. Yao Z, Yang S, He W, Li L, Xu R, Zhang X, Li H, Zhan R, Sun W, Tan J, et al: P311 promotes renal fibrosis via TGF $\beta 1 / \mathrm{Smad}$ signaling. Sci Rep 5: 17032, 2015.

33. Martin P: Wound healing - aiming for perfect skin regeneration. Science 276: 75-81, 1997.

34. Tomasek JJ, Gabbiani G, Hinz B, Chaponnier C and Brown RA: Myofibroblasts and mechano-regulation of connective tissue remodelling. Nat Rev Mol Cell Biol 3: 349-363, 2002.

35. Allison SJ: Fibrosis: Targeting EMT to reverse renal fibrosis. Nat Rev Nephrol 11: 565, 2015.

36. O'Connor JW and Gomez EW: Biomechanics of TGF $\beta$-induced epithelial-mesenchymal transition: Implications for fibrosis and cancer. Clin Transl Med 3: 23, 2014.

37. Wei MG, Sun W, He WM, Ni L and Yang YY: Ferulic acid attenuates TGF- $\beta 1$-induced renal cellular fibrosis in NRK-52E cells by inhibiting Smad/ILK/Snail pathway. Evid Based Complement Alternat Med 2015: 619720, 2015 